\title{
Effet d'une carence hydrique sur certains aspects du métabolisme phosphocalcique d'un rongeur semi-désertique (Meriones shawi) en croissance
}

\author{
Malika SAHNI, Jacqueline PEIGNOUX-DEVILLE, Evelyne LOPEZ, Francine LAL- \\ LIER, Elisabeth MARTELLY, Bernadette VIDAL \\ Laboratoire de Physiologie générale et comparée \\ du Muséum National d'Histoire Naturelle et \\ Laboratoire d'Endocrinologie comparée associé au C.N.R.S., \\ 7, rue Cuvier, 75231 Paris Cedex 05.
}

Summary. Effects of prolonged water deficiency on some aspects of phosphocalcic metabolism in a growing sub-desert rodent, Meriones shawi.

The effects of prolonged water deficiency on osseous tissue and phosphocalcic metabolism were studied in growing sub-desert rodents (Meriones shawi). The animals were progressively deprived of water since weaning. Throughout the experimental period we measured plasma levels of calcium and inorganic phosphate; in adults we used histomorphometric methods to estimate trabecular bone volume (TBV), bone mass, trabecular osteoid volume (TOV), osteoblasts (\%), osteoclasts (\%) and the trabecular resorption surfaces (TRS) characterizing the remodelling process of spongious and compact bone. The effect of dehydration on body weight and food consumption was also studied.

There were no significant differences in the plasma levels of either calcium or inorganic phosphate between the two groups.

On the other hand, in the dehydrated meriones we found wide differences in bone structure and remodelling (compared to the controls) which included a decrease in the cortical thickness of compact bone and a reduction of the spongious bone mass. These changes might be explained partly by the increase in osteoclastic resorption. The processes of bone formation were also greatly altered, as expressed in spongious bone by the accumulation of osteoid tissue and in compact bone by differences in the orientation of the bone layers.

\section{Introduction.}

L'aridité des régions désertiques constitue souvent un facteur limitant pour la croissance et la survie des espèces qui les peuplent. Les répercussions de la carence hydrique ont été souvent étudiées sur le métabolisme de l'eau et la répartition des liquides corporels, chez le mouton Chokla (Taneja, 1965 ; Purohit et al., 1972), le chameau (Schmidt-Nielsen, 1964) et le psammomys un Gerbillidé semidésertique (Ben Chaoucha-Chekir et al., 1983). More et Sahni (1979) ont étudié l'effet à long terme de la privation d'eau saisonnière chez le mouton Chokla ; celui-ci subit une perte de $16,4 \%$ de poids en été, accompagnée d'une économie 
d'eau de 30 à $40 \%$. Plusieurs auteurs ont étudié l'influence de la température sur la croissance et le métabolisme phosphocalcique chez le rat (Lee et al., 1969 ; Brown et Hacker, 1974 ; Al Hilli et Wright, 1983), mais peu d'entre eux considèrent ces facteurs après déshydratation. Pour Libermann et al. (1976) le rat adulte soumis à une carence hydrique aiguë ( 3 à 9 jours) montre une diminution du volume d'eau plasmatique proportionnelle à la chute d'eau corporelle totale ; il s'en suit une diminution de la circulation du calcium et du phosphate inorganique, mais en aucun cas il ne s'agit d'un changement de leurs concentrations plasmatiques. Au laboratoire nous avons constaté que la mérione (Mériones shawi), Gerbillidé d'Afrique du Nord, au stade adulte, pouvait supporter un régime dépourvu d'eau pendant au moins 6 mois. Cet animal constitue donc un modèle biologique intéressant pour l'étude d'une carence hydrique chronique sur différents paramètres du métabolisme phosphocalcique.

Les animaux étant privés d'eau dès leur sevrage, l'évolution du poids corporel, de la calcémie et de la phosphatémie a été étudiée pendant leur croissance ; puis au stade adulte, chez les mêmes animaux, ont été évaluées les éventuelles modifications pouvant affecter le fonctionnement et la structure de l'os compact et spongieux.

\section{Matériel et méthodes.}

De jeunes mériones (Meriones shawi shawi) âgées de 25 jours (poids moyen $=23,4 \pm 0,4 \mathrm{~g}$ ) ont été, aussitôt après le sevrage, isolées en chambre climatique $\left(\mathrm{T}^{\mathrm{O}}=25^{\circ} \mathrm{C}, \mathrm{Hr}=65 \%\right.$, photopériode $\left.12 \mathrm{~L}-12 \mathrm{D}\right)$ dans des cages individuelles $(20 \times 19 \times 15 \mathrm{~cm})$. Au fur et à mesure des naissances, chaque fratrie a été soumise à l'un ou l'autre des régimes décrits ci-dessous jusqu'à obtention de deux groupes de 20 animaux chacun. Tous les animaux ont été sacrifiés en même temps lorsqu'ils avaient 3 à 4 mois. Au moment du sevrage les animaux des deux régimes ont reçu une injection intrapéritonéale de $0,05 \mathrm{mg} / \mathrm{g}$ de poids d'achromycine $\mathrm{V}$ Lederle de façon à marquer, au niveau osseux, le début de l'un ou l'autre des régimes.

Les animaux témoins ont reçu un régime alimentaire constitué de biscuits pour rats (aliment complet stérilisable U.A.R.) et de pommes ad libitum (les pommes étant leur unique source d'eau) jusqu'au sacrifice.

Les animaux "déshydratés " ( $\left.{ }^{1}\right)$ ont reçu un régime, dans un premier temps identique à celui des témoins, puis l'apport en pommes a été réduit progressivement. Les quantités de pommes fournies ont été déterminées d'après le calcul des besoins en eau de la mérione selon la formule établie par Nicol (1978) sur les Euthériens et modifiée pour les mériones en croissance. Cette formule a permis de déterminer, chez la mérione en croissance, la quantité de pommes (donc l'eau, la pomme en contient environ $90 \%$ ) nécessaire à son développement : celle-ci est de $8 \mathrm{~g}$ pour les animaux témoins pesant en moyenne $24 \mathrm{~g}$. De façon à adapter progressivement la mérione à un régime sans eau, nous lui avons fourni, pendant les 3 premières

(1) Le terme " déshydraté " est utilisé pour : soumis progressivement à une carence hydrique. 
semaines de l'expérience, la moitié de la quantité de pommes qui lui est nécessaire, puis le quart pendant 2 semaines, ensuite l'apport en pommes a été supprimé complètement jusqu'à la fin de l'expérience. Les teneurs en calcium ( $\mathrm{Ca}$ ) et en phosphate inorganique ( $\mathrm{Pi}$ ) ont été déterminées dans les biscuits et les pommes.

Chaque animal recevant l'un ou l'autre des deux régimes est pesé à $0,1 \mathrm{~g}, 3$ fois par semaine, jusqu'à la fin de l'expérience.

Notons que les mériones déshydratées manifestent une activité et une agressivité accrues par rapport aux animaux témoins.

Les prélèvements sanguins ont été effectués tous les 15 jours, sur l'animal non anesthésié, par ponction dans le sinus infra-orbitaire.

Une semaine avant le sacrifice, la consommation alimentaire en pommes et en biscuits a été estimée à l'aide de ${ }^{22} \mathrm{Na}$ par la méthode de Green (1978). Ces valeurs ainsi que les teneurs en $\mathrm{Ca}$ et $\mathrm{Pi}$ des aliments ont permis le calcul de l'apport journalier en $\mathrm{Ca}$ et $\mathrm{Pi}$ chez les témoins et les déshydratés.

Le dosage du calcium plasmatique et alimentaire a été réalisé par spectrophotométrie d'absorption atomique et celui du phosphate par colorimétrie. Le dosage du sodium ( $\mathrm{Na}$ ) alimentaire a été effectué par photométrie de flamme.

En fin d'expérience, les mériones ont été pesées puis sacrifiées. Les fémurs des deux membres ont été prélevés, la partie distale du fémur a été séparée de la partie diaphysaire.

Les échantillons osseux, sans déminéralisation préalable, ont été fixés et colorés simultanément dans de la fuchsine basique à $1 \%$ dans de l'alcool à $70 \%$ (Frost, 1959), déshydratés dans des solutions alcooliques à $1 \%$ de fuchsine basique. Après éclaircissement dans le toluène, les échantillons ont été inclus dans du méthacrylate de méthyle selon la méthode de Baud et Morgenthaler (1952). Les coupes ont été réalisées perpendiculairement à l'axe de l'os dans la tranche diaphysaire et parallèlement à cet axe dans la partie distale pour l'étude de la métaphyse.

Les mesures morphométriques ont été effectuées sur des coupes de $15 \mu$ d'épaisseur. Dans l'os compact, l'épaisseur corticale moyenne (e.c.m.) a été déterminée en mesurant la moyenne des longueurs de 10 segments de droites tracés perpendiculairement à la surface corticale et répartis sur l'ensemble de la section. Dans l'os métaphysaire spongieux, ont été mesurés: le volume trabéculaire osseux (VTO), ses deux composantes (la masse osseuse calcifiée et le volume de l'ostéoïde), le pourcentage des ostéoblastes, ainsi que les surfaces de résorption et le pourcentage des ostéoclastes. Les mesures ont été effectuées selon la méthode de Frost et al. (1962) reprise par Bordier et al. (1964), sur 4 coupes par animal à raison de 6 champs par coupe. Toutes les mesures ont été réalisées en suivant une ligne droite horizontale située toujours à la même distance du cartilage de croissance.

Pour les statistiques, une analyse de type non paramétrique (test de Fischer) a été appliquée aux résultats.

\section{Résultats.}

L'analyse de la courbe pondérale des animaux témoins (fig. 1) montre une augmentation puis une stabilisation aux environs de 95 jours. La courbe de poids des 
animaux " déshydratés » est pratiquement parallèle à celle des témoins jusqu'à l'âge de 62 jours. La carence partielle en eau ne semble pas modifier la vitesse de croissance. Puis la privation totale d'eau entraîne une chute pondérale de $10 \%$ $(p<0,2)$ en 4 jours. On constate ensuite une stabilisation du poids pendant 24 jours suivie d'une augmentation pondérale de $18 \%(p<0,05)$ en 4 jours. A ce moment la courbe de poids des mériones « déshydratées » tend à rejoindre celle des animaux témoins.

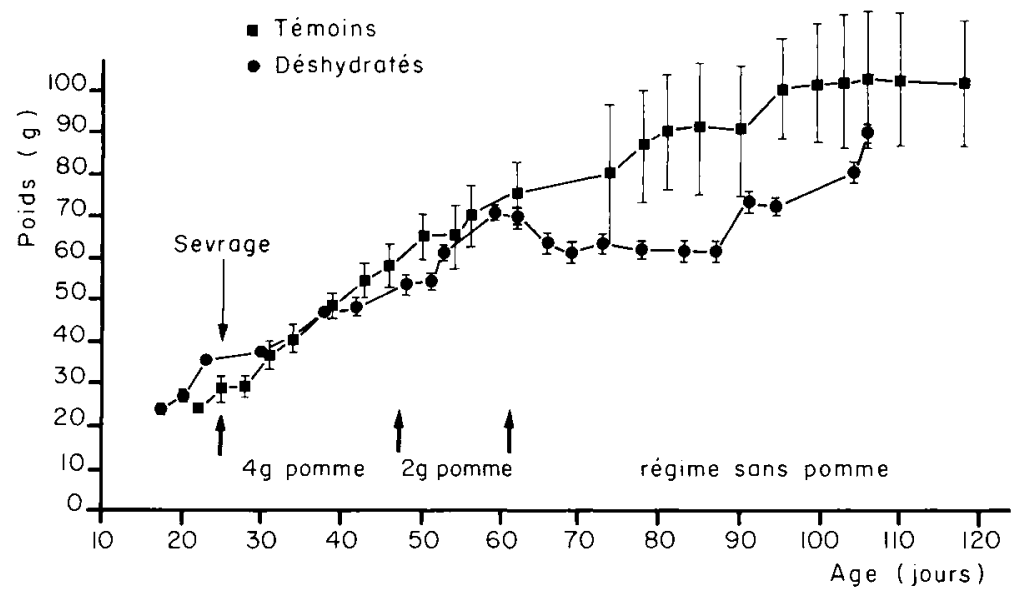

FIG. 1. - Evolution du poids des mériones témoins et "déshydratées " en fonction du temps. Chaque point représente la moyenne de 4 déterminations (correspondant à une fatrie). Nous avons choisi de montrer les courbes de poids d'une fatrie dans chacun des régimes, celles-ci étant représentatives de l'évolution pondérale dans toutes les autres fratries.

L'étude de la consommation alimentaire effectuée en fin d'expérience montre qu'à ce moment là, les mériones « déshydratées » consomment deux fois plus de biscuits que les témoins. De plus, l'apport journalier de calcium et de phosphate est plus important chez les mériones soumises à la déshydratation que chez les témoins (tabl. 1 et 2).

Les valeurs de calcémie et de phosphatémie déterminées tout au long de l'expérience ne montrent aucune variation significative, ni chez les animaux

\section{TABLEAU 1}

Teneur en calcium et en phosphate inorganique du régime alimentaire des mériones.

\begin{tabular}{lcc}
\hline $\begin{array}{l}\text { Eléments } \\
\text { (Poids frais) }\end{array}$ & Biscuits & Pommes \\
\hline $\begin{array}{l}\text { Calcium } \\
\mathrm{m} . \mathrm{mol} \cdot \mathrm{g}^{-1}\end{array}$ & 0,14 & 0,005 \\
\hline $\begin{array}{l}\text { Phosphate inorganique } \\
\mathrm{m} . \mathrm{mol}^{-1}\end{array}$ & 0,046 & 0,00046 \\
\hline
\end{tabular}


TABLEAU 2

Consommation alimentaire et apport en calcium et en phosphate chez les mériones témoins et " déshydratées ».

\begin{tabular}{|c|c|c|c|c|}
\hline & \multicolumn{2}{|c|}{$\begin{array}{l}\text { Consommation en } \\
\text { pommes et en biscuits }\end{array}$} & \multirow{2}{*}{$\begin{array}{c}\begin{array}{c}\text { Apport en } \\
\text { calcium }\end{array} \\
\mathrm{m} \mathrm{mol.} \mathrm{kg}^{-1} \mathrm{j}^{-1}\end{array}$} & \multirow{2}{*}{$\begin{array}{c}\begin{array}{c}\text { Apport en } \\
\text { phosphate }\end{array} \\
\mathrm{m} \mathrm{mol.} \mathrm{kg}^{-1} \mathrm{j}^{-1}\end{array}$} \\
\hline & $\begin{array}{l}\text { Pommes en } \\
\text { g. kg. }{ }^{-1} \mathrm{j}^{-1}\end{array}$ & $\begin{array}{l}\text { Biscuits en } \\
\text { g. } \mathrm{kg}^{-1} \mathrm{j}^{-1}\end{array}$ & & \\
\hline $\begin{array}{l}\text { Régime normal } \\
n=5 \\
(59)\end{array}$ & $345,5 \pm 23,2$ & $24,4 \pm 7,3$ & $5,1 \pm 0,1$ & $1,3 \pm 0,3$ \\
\hline $\begin{array}{l}\text { Régime déshydraté } \\
n=4 \\
(4 q)\end{array}$ & * & $51,6^{*} \pm 4,4$ & $7,1 \stackrel{*}{*} \pm, 6$ & $3,0 \pm 0,2$ \\
\hline
\end{tabular}

${ }^{*} \mathrm{p}<0,02$.

témoins, ni chez les animaux " déshydratés » (fig. 2 et fig. 3). Les valeurs moyennes de la calcémie et de la phosphatémie chez les témoins et chez les "déshydratés " sont respectivement égales à $(110,3 \pm 0,31 \mathrm{mg}(\mathrm{Ca}) / / ; 113,36 \pm 1,31 \mathrm{mg}(\mathrm{Ca}) / /$, avec $p>0,2)$ et $(96,31 \pm 0,54 \mathrm{mg}(\mathrm{Pi}) / \mathrm{l} ; 99,76 \pm 0,12 \mathrm{mg}(\mathrm{Pi}) / \mathrm{l}$, avec $\mathrm{p}>0,2)$. Elles ne présentent aucune différence significative.

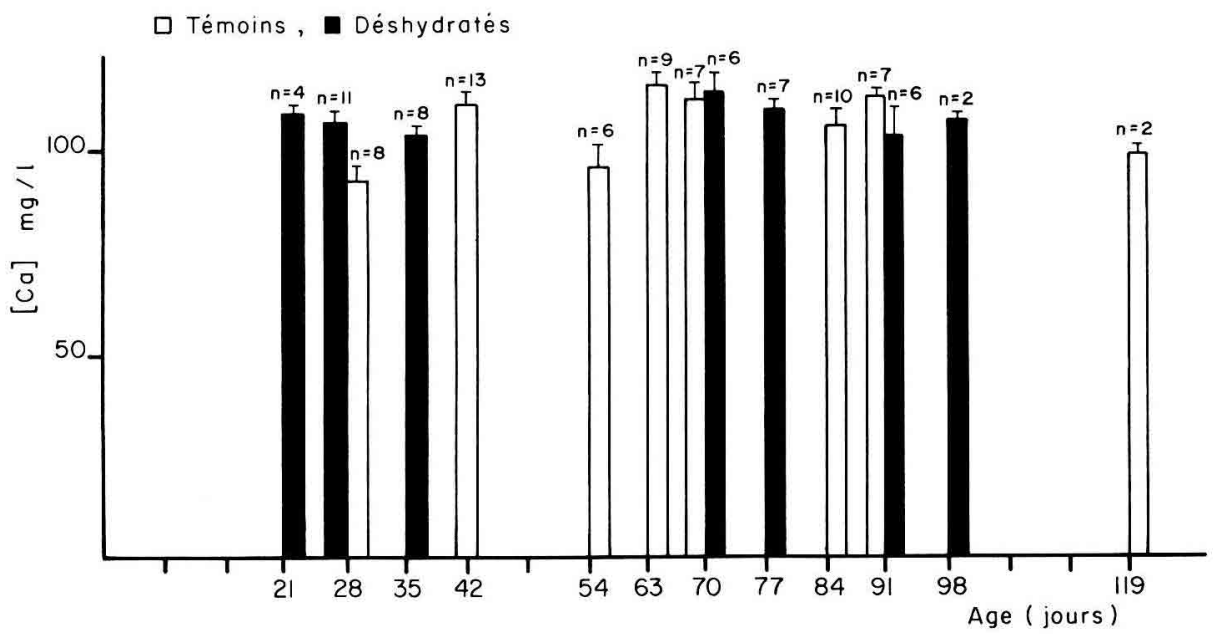

FIG. 2. - Evolution de la calcémie des mériones témoins et " déshydratées " en fonction du temps. $\mathrm{n}$ : nombre d'échantillons par groupe.

L'hématocrite reste constant dans chaque groupe au cours de la croissance et les valeurs moyennes calculées chez les témoins $(48,71 \pm 1,7 \%)$ et chez les " déshydratés" $(48,06 \pm 1,30 \%)$ ne montrent pas de différence significative (p $>0,2)$.

L'observation des coupes frontales des métaphyses fémorales indique chez les animaux " déshydratés » l'existence de travées osseuses, constituant l'os spon- 


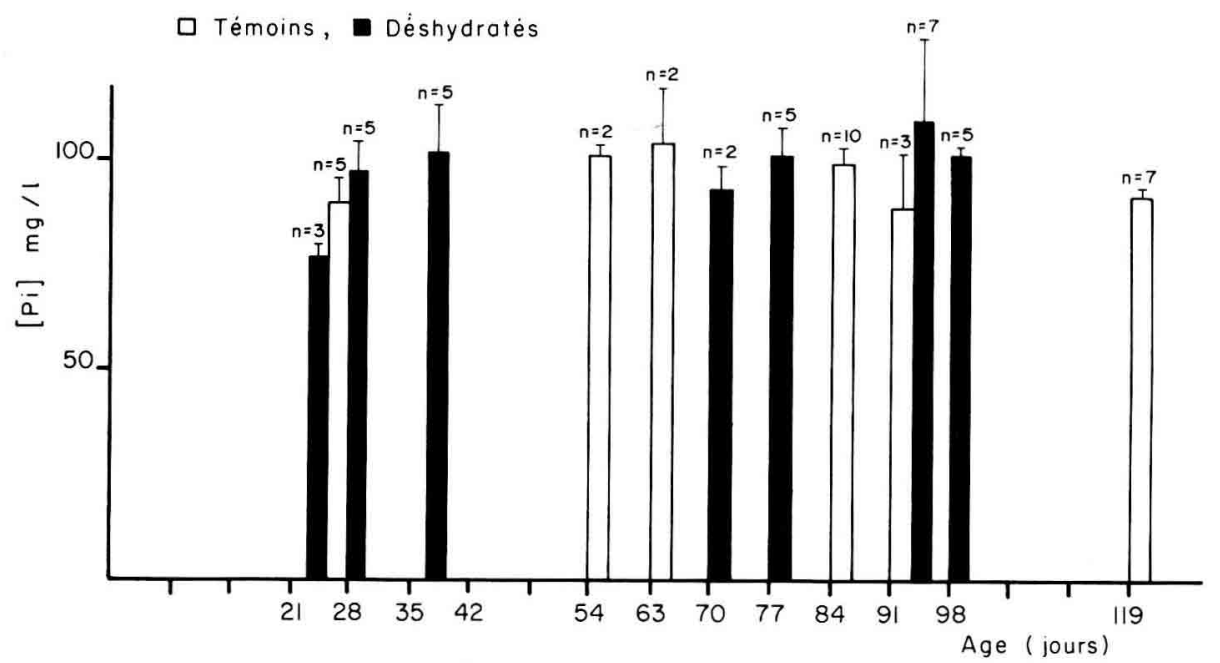

FIG. 3. - Evolution de la phosphatémie des mériones témoins et "déshydratées » en fonction du temps $n$ : nombre d'échantillons par groupe.

gieux, moins importantes que chez les témoins (fig. 4, fig. 5). Ces travées sont bordées, par endroits, par une couche d'os non minéralisé appelé couramment ostéoïde (fig. 6, fig. 7). De plus, des ostéoclastes sont présents en abondance dans l'os spongieux métaphysaire des mériones soumises à une carence hydrique (tabl. 3) ; cette présence de cellules ayant une fonction résorptive s'accompagne de surfaces de résorption fortement colorées à la fuchsine basique (fig. 5).

L'os compact diaphysaire des animaux " déshydratés " présente aussi de nombreux ostéoclastes enfouis dans des lacunes de Howship (fig. 9). L'abondance et l'aspect actif de ces cellules, souvent plurinucléées et en contact avec une surface de résorption colorée à la fuchsine basique, traduisent une importante activité résorptive au niveau périostique qui n'apparaît pas sur les coupes diaphysaires des animaux témoins (fig. 8). Dans l'état actuel de nos travaux, cette résorption diaphysaire n'a pas encore été quantifiée. Des modifications de structure dans l'os compact ont été constatées. En effet, à l'observation en lumière transmise, l'organisation en ostéones primaires semble différente chez les mériones « déshydratées » de celle mise en évidence chez les témoins. L'observation en lumière polarisée indique une biréfringence moins forte des lames osseuses ; de plus, ces lames osseuses qui,

FIG. 4. - Coupe frontale de métaphyse fémorale de mérione témoin montrant certaines travées osseuses $(\rightarrow$ ). Coloration fuchsine basique. $\times 250$.

FIG. 5. - Coupe frontale de métaphyse fémorale de mérione " déshydratée ». Noter la faible importance de la masse osseuse $(\rightarrow$ ) formant les travées ainsi que la présence d'ostéoclastes $1 \vec{\nabla} \mid$ situés dans des lacunes de résorption. $\times 250$.

FIGS 6 et 7. - Coupes frontales de métaphyses fémorales de mérione. Comparer l'épaisseur de l'ostéoïde $(\rightarrow$ ) bordant les travées osseuses chez une mérione témoin (fig. 6) avec celle observée $(\rightarrow$ ) chez une mérione déshydratée (fig. 7$) . \quad \times 250$. 

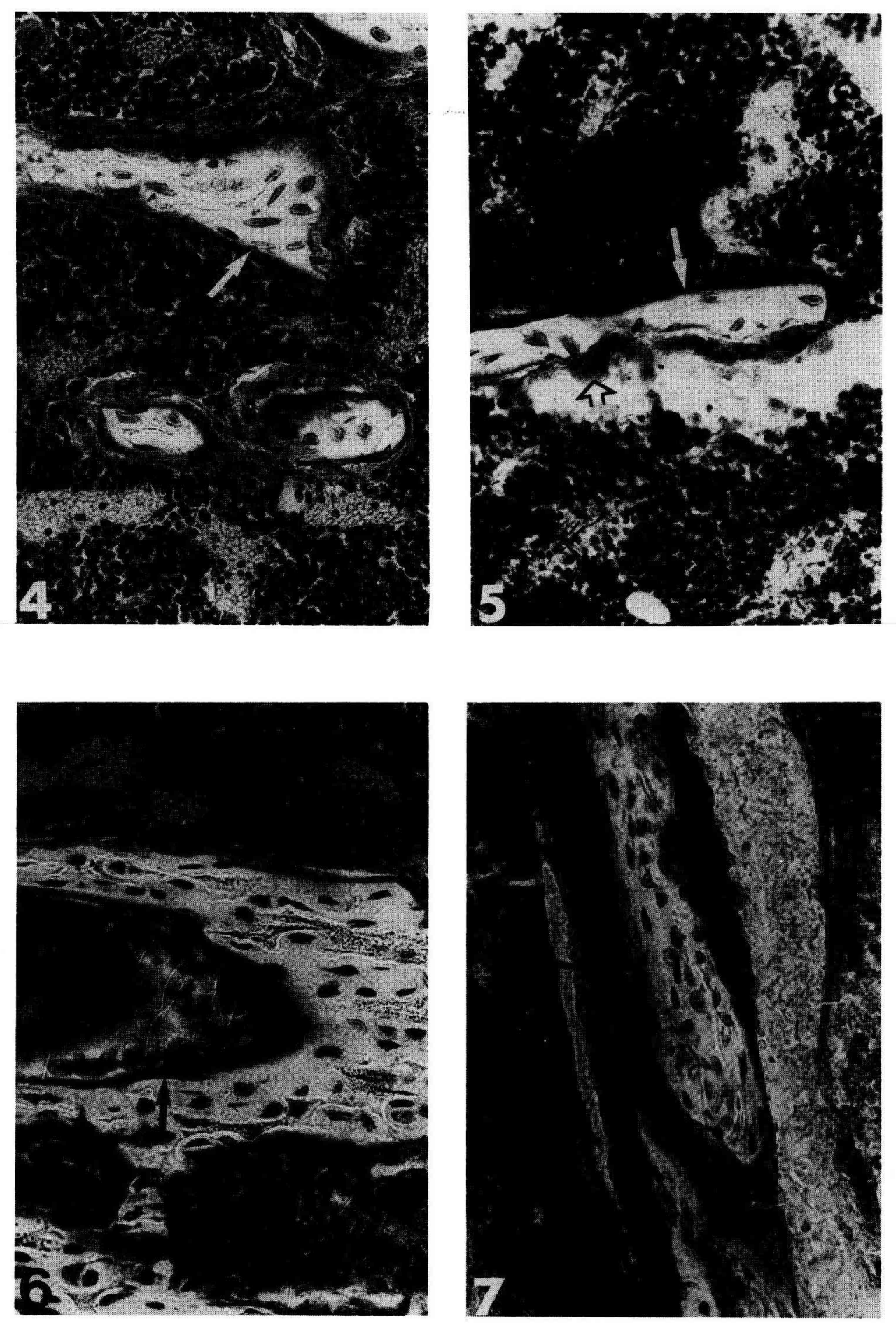
dans la diaphyse fémorale des témoins sont organisées en couches concentriques autour des canaux vasculaires, ne présentent aucune orientation préférentielle chez les animaux carencés en eau.

L'étude histomorphométrique de l'os spongieux et de l'os compact montre dans l'os spongieux (tabl. 3) une diminution hautement significative du volume trabéculaire osseux chez les animaux " déshydratés ". II n'y a aucune différence de pourcentage des ostéoblastes entre les animaux soumis aux deux régimes (tabl. 3)

\section{TABLEAU 3}

Comparaison de différents paramètres de l'os spongieux chez des mériones témoins et " déshydratées".

\begin{tabular}{|c|c|c|c|c|c|c|}
\hline & $\begin{array}{l}\text { VTO } \\
\text { (1) }\end{array}$ & $\begin{array}{c}\text { Volume } \\
\text { ostéoïde } \\
\text { (1) }\end{array}$ & $\begin{array}{c}\% \text { ostéoblastes } \\
\text { (2) }\end{array}$ & $\begin{array}{c}\text { Masse calcifiée } \\
\text { (1) }\end{array}$ & $\begin{array}{c}\text { \% ostéoclastes } \\
\text { (2) }\end{array}$ & $\begin{array}{l}\text { Surface de } \\
\text { résorption } \\
\text { (3) }\end{array}$ \\
\hline $\begin{array}{l}\text { Témoins } \\
n=7 \\
(5 \%, 2 \propto)\end{array}$ & $49,34 \pm 1,67$ & $8,25 \pm 0,44$ & $50 \pm 0,03$ & $40,04 \pm 1,45$ & $\pm 1,93$ & $3,05 \pm 1,57$ \\
\hline $\begin{array}{l}\text { "Déshydratés" } \\
n=6 \\
(39,3 \circ)\end{array}$ & $33,68 \pm 1,71$ & $16,25 \pm 1,58$ & $47 \pm 0,05$ & $17,20 \pm 1,47$ & $62,00 \pm 5,43$ & $\underset{14,96 \pm 2,58}{\Delta}$ \\
\hline
\end{tabular}

* $p<0,001 ; \Delta \mathrm{A}<0,01 ; \bullet p>0,02$

(1) : en \% de la masse osseuse métaphysaire ; (2) : par rapport à la surface osseuse ; (3) : en \% de la surface totale de la masse osseuse spongieuse.

et la mesure du volume de l'ostéoïde met en évidence une nette augmentation de ce paramètre chez les mériones " déshydratées ". En conséquence, il y a diminution de la masse calcifiée chez les animaux carencés en eau. De plus chez ces derniers, nous observons une augmentation hautement significative de la résorption ostéoclastique et du pourcentage des ostéoclastes. Dans l'os compact, les mesures de l'épaisseur corticale moyenne diaphysaire (tabl. 4) mettent en évidence une diminution de celle-ci chez les mériones carencées en eau. L'examen des coupes non colorées en lumière ultraviolette montre qu'avant le sevrage et avant le début de l'adaptation au régime déshydraté, la quantité d'os apposée aussi bien dans le cas des animaux témoins que " déshydratés " est la même alors qu'après le sevrage (marquée

FIG. 8. - Coupe transversale de diaphyse fémorale de mérione témoin. Noter l'organisation des ostéones primaires et la surface périostique à bords lisse $(\longrightarrow) \times 250$.

FIG. 9. - Coupe transversale de diaphyse fémorale de mérione " déshydratée ". Noter l'absence de couches osseuses concentriques autour des canaux vasculaires et la présence de nombreux ostéoclastes $(\rightarrow$ ) à la surface du périoste avec surface de résorption. $\times 250$.

FIG. 10. - Coupe transversale de diaphyse fémorale de mérione témoin, marquée par la tétracycline, montrant la quantité d'os déposée, après le sevrage $(-4) . \times 63$.

FIG. 11. - Coupe transversale de diaphyse fémorale de mérione "déshydratée " marquée par la tétracycline montrant la réduction de la quantité d'os totale déposée durant la vie de l'animal due à un moindre dépôt après la déshydratation $(>4) \times 63$. 

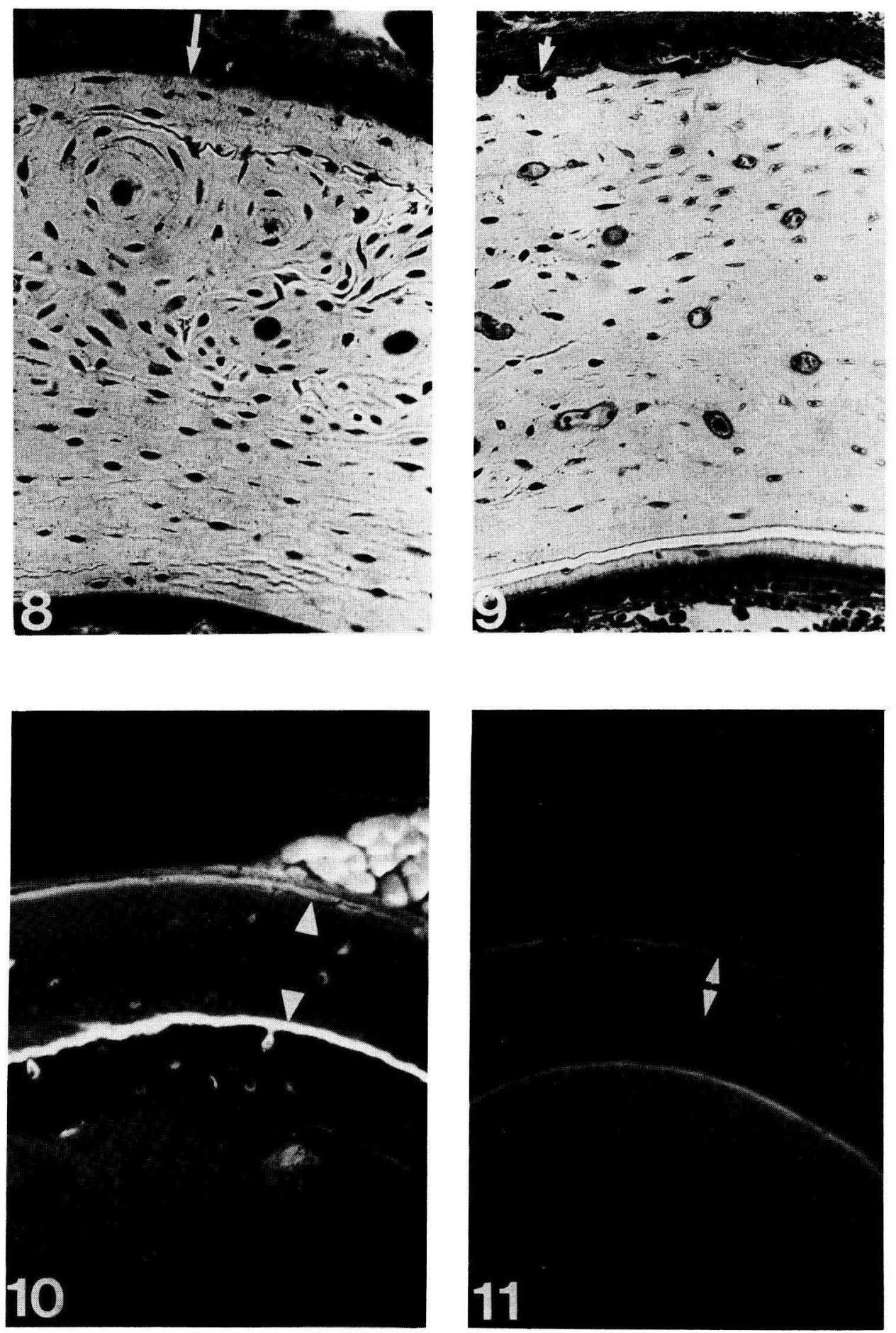
par un trait fluorescent), la quantité d'os apposée chez les animaux « déshydratés » est nettement inférieure à celle observée chez les témoins (fig. 10, fig. 11).

\section{TABLEAU 4}

Epaisseur corticale moyenne (e.c.m.) chez les mériones témoins et " déshydratées ».

\begin{tabular}{|c|c|}
\hline & Moy. (e.c.m.) $\mu \mathrm{m}$ \\
\hline $\begin{array}{c}\text { Témoins } \\
n=5 \\
(3 q, 2 \circ)\end{array}$ & $422,76 \pm 29,63$ \\
\hline $\begin{array}{c}\text { Déshydratés } \\
n=9 \\
\left(6 \%, 3 \sigma^{\prime}\right)\end{array}$ & $250,82 \pm 17,00$ \\
\hline
\end{tabular}

${ }^{*} p<0,001$

\section{Discussion.}

Les mériones en cours de croissance sont capables de résister à une carence hydrique prolongée et imposée dès leur sevrage. Cette carence hydrique n'est cependant pas sans retentissement sur l'évolution du poids corporel des animaux. Elle provoque des modifications de la consommation alimentaire, de la structure et du remaniement du tissu osseux. La mesure de la consommation alimentaire et le calcul de l'apport nutritionnel en calcium et en phosphate montrent que les mériones " déshydratées » ne présentent pas de carence en calcium ni en phosphate. Cependant le pourcentage de résorption est augmenté chez ces animaux.

La diminution de la masse osseuse dans l'os spongieux et compact des mériones soumises à une carence hydrique peut s'expliquer par l'augmentation du taux de résorption et par le défaut de minéralisation. Dans l'os spongieux l'accentuation des phénomènes de résorption et l'augmentation du pourcentage des ostéoclastes peuvent s'expliquer par l'intervention probable de l'hormone parathyroïdienne (PTH) hormone qui, chez les mammitères, libère le calcium en stimulant la résorption ostéoclastique (Barnicot, 1945 ; Chang, 1951 ; Gaillard, 1955). D'ailleurs chez le dromadaire soumis à déshydratation on constate une augmentation de l'activité parathyroidienne (Charnot, 1963).

Dans l'os compact, la diminution de l'épaisseur corticale diaphysaire est probablement due en partie à l'accroissement de la résorption ostéoclastique sous périostée observée mais surtout à une réduction de la formation osseuse nettement visible grâce au marquage par la tétracycline. Les observations effectuées dans l'os compact sur la différence d'orientation des couches osseuses, autour des canaux vasculaires, ainsi que leur faible biréfringence laissent supposer l'existence d'une perturbation, dans un premier temps de la formation de la matrice organique et dans un second temps des processus de dépôt du minéral.

II est généralement admis que l'accumulation d'ostéoïde le long des travées osseuses de l'os spongieux peut être le résultat de plusieurs phénomènes, soit d'une forte accentuation de l'apposition ostéoblastique ou d'un défaut de minéralisation 
comme dans les cas d'ostéomalacie (Ham et Cormack 1979). Eastwood et al. (1974) ont démontré que la constance du produit des taux de $(\mathrm{Pi} \times \mathrm{Ca}$ ) plasmatiques n'est pas suffisante pour la réalisation d'un processus de minéralisation normal, dans lequel intervient le $\mathrm{Pi}$ et les métabolites de la vitamine $\mathrm{D}$. De même une carence en Vit $D$ chez le rat entraîne un défaut de minéralisation se traduisant essentiellement par une augmentation du tissu ostéoïde (Underwood et al., 1951). Le pourcentage des ostéoblastes n'étant pas différent entre les mériones témoins et " déshydratées ", l'accentuation du volume de l'ostéoïde pourrait s'expliquer par un défaut de minéralisation.

L'hypertrophie rénale observée chez les mériones carencées en eau (Sahni et al., en préparation) laisse supposer l'existence de modifications profondes au niveau du rein, susceptibles d'entraîner des troubles de la réabsorption tubulaire du calcium et du phosphate, éléments indispensables à une minéralisation complète. Le déséquilibre rénal supposé pourrait entraîner un défaut de formation de l'un des métabolites de la Vit $D_{3}$ en l'occurence de $1,25(\mathrm{OH})_{2}$ vit $D_{3}$ formé dans le rein par hydroxylation du $25(\mathrm{OH})$ vit $\mathrm{D}_{3}$ (Fraser, 1980), et ainsi expliquer, tout au moins en partie, certaines modifications osseuses observées.

Reçu en mars 1986.

Accepté en octobre 1986.

Remerciements. - Nous remercions Monsieur le Professeur C. A. Baud (Institut de Morphologie du Centre Médical Universitaire de Genève) qui nous a fait bénéficier de ses précieux conseils, Monsieur P. Lafay pour l'iconographie, ainsi que Madame F. Lieron pour la dactylographie de cet article.

Ce travail a été effectué grâce au contrat A.T.P.-C.N.R.S., "Réponse biochimique et physiologique à des situations vitales critiques $", n^{\circ} 960089$.

\section{Références}

AL HILLI F., WRIGHT E. A., 1983. The effects of changes in the environmental temperature on the growth of bone in the mouse. Radiological study. Br. J. exp. Path., 64, 43-52.

BARNICOT N. A., 1945. Some data on the effect of parathormone on the greylethal mouse. J. Anat., 79, 83-91.

BAUD C. A., MORGENTHALER P. W., 1952. Recherches sur l'ultrastructure de l'os humain fossile. Arch. Suisses Anthropol. Gen., 17, 52-65.

BEN CHAOUCHA-CHEKIR R., LACHIVER F., CHENITI T., 1983. Données préliminaires sur le taux de renouvellement d'eau chez un Gerbillidé désertique : Psammomys obesus étudié dans son environnement naturel en Tunisie. Mammalia, 47, 544-547.

BORDIER Ph., MATRAJT J., MIRAVET L., HIOCO D., 1964. Mesure histologique de la masse de la résorption des travées osseuses. Path. Biol., 12, 1238-1243.

BROWN R. G., HACKER R. R., 1974. Connective tissue metabolism in Swine. Influence of environmental temperature on calcium and phosphorus excretion and bone mineral composition. Growth, 38, 401-411.

CHARNOT Y., 1963. Premières observations sur les parathyroides de dromadaire. Bull. Soc. Sci. Nat. Phys. Maroc, 281-288.

CHANG H. Y., 1951. Grafts of parathyroid and other tissues to bone. Anat. Rec., 111, 23.

EASTWOOD J. B., BORDIER P. J., CLARKSON E. M., TUNCHOT S., De WARDENER H. E., 1974. The contrasting effects on bone histology of Vit. $D$ and of calcium carbonate in the osteomalacia of chronic renal failure. Clin. Sci. mol. Med., 47, 23-42. 
FRASER D. R., 1980. Regulation of the metabolism of vitamin D. Phys. Rev., 60, 551-613.

FROST M. M., 1959. Staining of fresh, undecalcified, thin bone section. Stain Technol., 34, 135-146.

FROST M. M., VILLANUEVA A. R., ROTH H., 1962. Qualitative method for measuring osteoclastic activity. Henry Fond. Hosp. Bull., 10, 217-228.

GAILLARD P. J., 1955. Parathyroid gland tissue and bone « in vitro ». I. Exp. Cel/ Res., suppl. 3. 154.

GREEN B., 1978. Estimation of food consumption in the dingo Canis familiaris dingo by means of ${ }^{22} \mathrm{Na}$ turnover. Ecology, 59, n० 2, 207-210.

HAM A. W., CORMACK D. H., 1979. Bone and bones, 377-462. In Histophysiology of cartilage, bone and joints. J. B. Lippincott Co.

LEE M. M. C., CHU P. C., CHAN H. C., 1969. Effects of cold on the skeletal growth of albino rats. Am. J. Anat., 124, 239-250.

LIBERMANN I. M., BRAZZAUNA H., RODRIGUEZ J., CAPANO A., NEUMARK J., 1976. Changes of plasma volume and plasma composition in water deprived rats. Eur. J. Physiol. Pfl. Arch., 365, 191-196.

MORE T., SAHNI L. K., 1979. Effects of water deprivation on growth and body fluid composition in chokla lambs under semi arid conditions. Indian J. anim. Sci., 48, 600-604.

NICOL S. C., 1978. Rates of water turnover in Marsupials and Eutherians : a comparative review with new data on the Tasmanian Devil. Aust. J. Zool., 26, 465-473.

PUROHIT S. R., GHOCH P. K., TANEJA G. C., 1972. Water metabolism in desert sheep : effects of various degrees of water restriction on the distribution of body water in Marwari sheep. Aust. J. agric. Res., 23, 605-691.

SCHMIDT-NIELSEN K., 1964. Desert animals physiological problems of heat and water. Clarenton Press, Oxford.

TANEJA G. C., 1965. Effects of varying frequency of watering during summer on the temperature respiration, body-weight and packed cell volume of blood of sheep. Indian J. exp. Biol., 4, 259262.

UNDERWOOD E., FISCH S., HODGE H. C., 1951. Metabolism of a calcium in normal, rachitic and Vit $\mathrm{D}$ treated rats and evidence by radio-calcium ${ }^{45} \mathrm{Ca}$ studies. Am. J. Physiol., 166, 387-393. 